\title{
眮
}

\section{A database for water transitions from experiment and theory}

Jonathan Tennyson

Department of Physics and Astronomy

University College London
HITRAN meeting

Harvard

June 2006

The Earth seen in water vapour by NASA's GOES satellite 


\section{Why water vapour?}

- Molecule number 1 in HITRAN

- Major (70\%) atmospheric absorber of incoming sunlight

- Even $\mathrm{H}_{2}{ }^{18} \mathrm{O}$ is fifth biggest absorber

- Largest (60\%) greenhouse gas

- Atmospheres of cool stars

- Combustion

- Life !? 


\section{UCL strategy for a reliable, complete (300K) linelist}

- Strong lines:

water-air spectra, variable path-length

- Weak lines:

water vapour spectra, longest path-length \& integration times possible

- Isotopologues:

Isotopically enhanced samples (Kitt Peak, CRDS)

- Completeness/assignments:

High quality variational calculations 


\section{IUPAC Task group}

A database of water transitions from experiment and theory

- Water lines at room temperature (HITRAN)

- Hot water

- Isotopologues

- Line profiles

- Theory

- Validation

- Database

Meet room P226 "Tea Room"

Weds from $2.30 \mathrm{pm}$

Thurs until lunch 


\section{Scope}

- transitions $0-30,000 \mathrm{~cm}^{-1}$.

- linelist for room temperature $(\mathbf{C}, 296 \mathrm{~K}) \&$ hot $(\mathbf{H})$ water.

- $\mathbf{C}$ complete for intensities $>10^{-29} \mathrm{~cm}$ molecule ${ }^{-1}$

\section{in natural abundance.}

- Singly \& doubly substituted isotopologues:

$\mathrm{HD}^{16} \mathrm{O}, \mathrm{H}_{2}{ }^{18} \mathrm{O}, \mathrm{H}_{2}{ }^{17} \mathrm{O}, \mathrm{D}_{2}{ }^{16} \mathrm{O}, \mathrm{HD}^{17} \mathrm{O}$, and $\mathrm{HD}^{18} \mathrm{O}$.

No triply substituted isotopologues, no tritium.

- Line profiles: function form?

Broadening parameters $\gamma$ and $\delta$.

Dependence: pressure $(0-3 \mathrm{~atm})$, temperature $(200-300 \mathrm{~K})$

Experimental \& computational data.

Parameters for self- , $\mathrm{N}_{2}, \mathrm{O}_{2}$, air, and $\mathrm{H}_{2}$ broadening. 


\section{Database}

- Master database to be prepared for each isotopologue.

- Should capture origin \& time-dependence of measured and computed values.

- Both 'old' and 'new' data archived and accessible.

- Flexible in terms of data structures

- HITRAN "button" 


\section{Master file strategy}

- Use most complete (not necessarily best) as Master file eg BT2

- Augment with data from other sources: expt, other theory

- Store all known data: use error analysis to combine

- Clear data history

- Files structured by function: levels, transitions (+ mixings?)

- Distributed data?

- Some functionality in-built eg HITRAN button 


\section{New BT2 linelist}

Barber et al, Mon. Not. R. astr. Soc. 368, 1087 (2006).

http://www.tampa.phys.ucl.ac.uk/ftp/astrodata/water/BT2/

- 50,000 processor hours.

- Wavefunctions $>0.8$ terabites

- 221,100 energy levels (all to $\mathrm{J}=50, \mathrm{E}=30,000 \mathrm{~cm}^{-1}$ ) 14,889 experimentally known

- 506 million transitions (PS list has 308m) $>100,000$ experimentally known with intensities

- $\rightarrow$ Partition function $99.9915 \%$ of Vidler \& Tennyson's value at $3,000 \mathrm{~K}$ 


\section{Comparison with Experimental Levels}

\begin{tabular}{|lcc|}
\hline & BT & PS \\
Agreement: & $\%$ & $\%$ \\
Within $0.10 \mathrm{~cm}^{-1}$ & 48.7 & 59.2 \\
Within $0.33 \mathrm{~cm}^{-1}$ & 91.4 & 85.6 \\
Within $1 \mathrm{~cm}^{-1}$ & 99.2 & 92.6 \\
Within $3 \mathrm{~cm}^{-1}$ & 99.9 & 96.5 \\
Within $5 \mathrm{~cm}^{-1}$ & 100.0 & 97.0 \\
Within $10 \mathrm{~cm}^{-1}$ & 100.0 & 98.1 \\
\hline
\end{tabular}




\section{Raw spectra from DVR3D program suite}

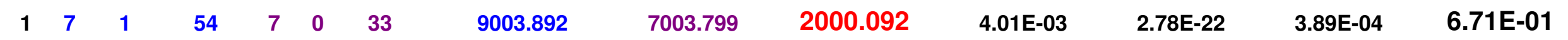

\begin{tabular}{|c|c|c|c|c|c|c|c|c|c|c|c|c|c|}
\hline 1 & 3 & 0 & 38 & 3 & 1 & 17 & 9098.530 & 7098.116 & 2000.415 & 1.56E-03 & 1.01E-22 & 1.41E-04 & 5.59E-01 \\
\hline 1 & 7 & 0 & 84 & 6 & 0 & 47 & 10486.138 & 8485.481 & 2000.657 & 4.69E-02 & 1.12E-21 & 1.56E-03 & 7.84E+00 \\
\hline 1 & 6 & 0 & 77 & 6 & 1 & 45 & 10939.532 & 8938.685 & 2000.848 & 4.83E-03 & 8.33E-23 & 1.16E-04 & 9.34E-01 \\
\hline 1 & 6 & 1 & 11 & 5 & 1 & 5 & 4407.221 & 2406.299 & 2000.922 & 2.77E-02 & 5.25E-20 & 7.34E-02 & 5.35E+00 \\
\hline C & 6 & 0 & 16 & 5 & 0 & 5 & 4407.355 & 2406.297 & 2001.058 & 3.26E-02 & 2.06E-20 & 2.88E-02 & 6.30E+00 \\
\hline
\end{tabular}

\begin{tabular}{|c|c|c|c|c|c|c|c|c|c|c|c|c|c|}
\hline 1 & 4 & 1 & 60 & 4 & 0 & 46 & 11384.245 & 9383.183 & 2001.062 & 6.66E-03 & 8.35E-23 & 1.17E-04 & 1.86E+00 \\
\hline 1 & 6 & 0 & 78 & 7 & 0 & 60 & 10955.914 & 8954.726 & 2001.188 & 1.69E-02 & 2.88E-22 & 4.03E-04 & 3.27E+00 \\
\hline 0 & 7 & 1 & 19 & 7 & 0 & 9 & 6034.992 & 4033.695 & 2001.297 & $7.29 \mathrm{E}-04$ & 1.43E-22 & $2.00 \mathrm{E}-04$ & 1.22E-01 \\
\hline 1 & 5 & 1 & 104 & 5 & 0 & 75 & 12912.871 & 10911.526 & 2001.344 & 3.36E-02 & $1.40 \mathrm{E}-22$ & 1.96E-04 & 7.68E+00 \\
\hline
\end{tabular}


Energy file: N

$\begin{array}{lllllll}E / c^{-1} & v_{1} & v_{2} & v_{3} & J & K_{a} & K_{c}\end{array}$

\begin{tabular}{|c|c|c|c|c|c|c|c|c|c|c|}
\hline A & B & C & D & E & $\mathbf{F}$ & $\mathbf{G}$ & H & $\mathbf{I}$ & $\mathbf{J}$ & K \\
\hline 43432 & 11 & 1 & 50 & 8730.136998 & 0 & 2 & 1 & 11 & 3 & 8 \\
\hline 43433 & 11 & 1 & 51 & 8819.773962 & 0 & 4 & 0 & 11 & 6 & 6 \\
\hline 43434 & 11 & 1 & 52 & 8918.536215 & 0 & 0 & 2 & 11 & 2 & 10 \\
\hline 43435 & 11 & 1 & 53 & 8965.496130 & 0 & 2 & 1 & 11 & 5 & 6 \\
\hline 43436 & 11 & 1 & 54 & 8975.145175 & 2 & 0 & 0 & 11 & 4 & 8 \\
\hline 43437 & 11 & 1 & 55 & 9007.868894 & 1 & 0 & 1 & 11 & 3 & 8 \\
\hline 43438 & 11 & 1 & 56 & 9082.413891 & 1 & 2 & 0 & 11 & 6 & 6 \\
\hline 43439 & 11 & 1 & 57 & 9170.343871 & 1 & 0 & 1 & 11 & 5 & 6 \\
\hline 43440 & 11 & 1 & 58 & 9223.444158 & 0 & 0 & 2 & 11 & 4 & 8 \\
\hline 43441 & 11 & 1 & 59 & 9264.489815 & 2 & 0 & 0 & 11 & 6 & 6 \\
\hline 43442 & 11 & 1 & 60 & 9267.088316 & 0 & 5 & 0 & 11 & 2 & 10 \\
\hline 43443 & 11 & 1 & 61 & 9369.887722 & 0 & 2 & 1 & 11 & 7 & 4 \\
\hline 43444 & 11 & 1 & 62 & 9434.002547 & 0 & 4 & 0 & 11 & 8 & 4 \\
\hline 43445 & 11 & 1 & 63 & 9457.272655 & 1 & 0 & 1 & 11 & 7 & 4 \\
\hline 43446 & 11 & 1 & 64 & 9498.012728 & 0 & 0 & 2 & 11 & 6 & 6 \\
\hline 43447 & 11 & 1 & 65 & 9565.890023 & 1 & 2 & 0 & 11 & 8 & 4 \\
\hline
\end{tabular}




\section{Transitions file:}

\begin{tabular}{|c|c|c|}
\hline 144848 & 146183 & 3.46E-04 \\
\hline 115309 & 108520 & 7.42E-04 \\
\hline 196018 & 198413 & 1.95E-04 \\
\hline 7031 & 7703 & 1.13E-02 \\
\hline 149176 & 150123 & 1.69E-04 \\
\hline 81528 & 78734 & 2.30E-01 \\
\hline 80829 & 78237 & 8.83E-04 \\
\hline 209672 & 210876 & $2.51 \mathrm{E}-01$ \\
\hline 207026 & 203241 & 2.72E-04 \\
\hline 188972 & 184971 & 1.25E-01 \\
\hline 152471 & 153399 & 1.12E-02 \\
\hline 39749 & 37479 & 1.46E-07 \\
\hline 10579 & 15882 & 6.90E-05 \\
\hline 34458 & 35617 & 1.15E-03 \\
\hline
\end{tabular}

$12.8 \mathrm{~Gb}$

Divided into

16 files by frequency

For downloading 


\section{Master file strategy: \\ Inclusion of Experimental (+ other theoretical) data}

Added to record. Data classified:

Property of level $\rightarrow$ Energy File

- Experimental levels (already included)

- Alternative quantum numbers (local modes)

Property of transition $\rightarrow$ Transition File

- Measured intensities or A coefficients

- Line profile parameters

Line mixing as a third file? 
Linelists available for Master databases

Author Potenial

$\mathrm{H}_{2}{ }^{16} \mathrm{O}$

BT2

$\mathrm{H}_{2}{ }^{17} \mathrm{O}$ Shirin

$\mathrm{H}_{2}{ }^{18} \mathrm{O}$ Shirin

FIS3 (2006)

HDO Tashkun

$\mathrm{D}_{2}{ }^{16} \mathrm{O} \quad$ Zobov

$\mathrm{HD}^{18} \mathrm{O}$

$\mathrm{HD}^{17} \mathrm{O}$

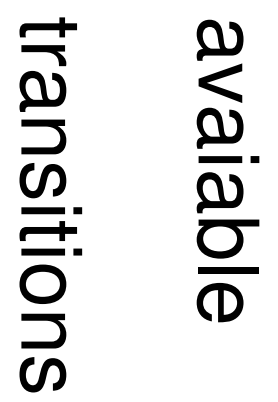

50

FIS3 (2006) 26000? 10

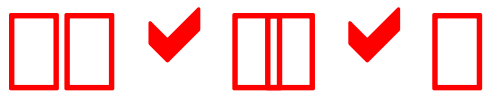

$\square \square \vee \mathbb{U} \vee \square$

10

$26000 ?$

30
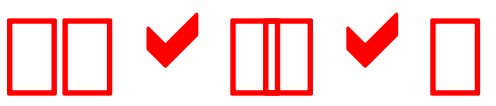

$\square \square$

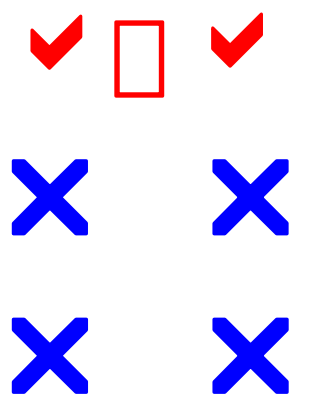




\section{Main characteristics (poster by Attila Csaszar)}

- Dual database of rovibrational energy levels and rovibrational transition with well-defined uncertainties

- Complete collection and storage of all relevant spectroscopic data for all major isotopologues of water

- Critical evaluation of data which will always carry their own pedigree (e.g., bibliographical references, important measurement conditions, metadata)

- Inclusion of intensities, line widths, and line broadenings in the database, possibly including refinement of relevant parameters

- Global multi-dataset optimization

- Curation, organizational, data-mining and displaying tools

- Allow immediate (and automatic) consistency analysis of newly reported data before data deposition

- Allow „experiments” with what-if scenarios (important in order to predict what extra information new experiments might provide

- All supporting programs written in $\mathrm{C}++$ and Java

- Sensitivity analysis of uncertainties

- Reproduce all known and well-defined experimental data (time-dependence)

- Predictions are rigorously quantified by their respective uncertainty bounds

- Minimal chance of leaving feasible regions of parameters

- HITRAN „button” to produce the best available data in HITRAN form for modeling studies 


\section{IUPAC Task group}

A database of water transitions from experiment and theory MEMBERS:

Peter Bernath (Waterloo, Canada); Alain Campargue (Grenoble, France); Michel Carleer (Brussels, Belgium); Attila Császár (Budapest, Hungary); Robert Gamache (Lowell, U.S.A.); Joseph Hodges (NIST, U.S.A.); Alain Jenouvrier (Reims, France); Olga Naumenko (Tomsk, Russia); Oleg Polyansky (Ulm, Germany); Laurence Rothman (Harvard, U.S.A.); Jonathan Tennyson (London, U.K.); Robert Toth (JPL, U.S.A.); Ann Vandaele (Brussels, Belgium); Nikolai Zobov (Nizhny Novgorod, Russia) 


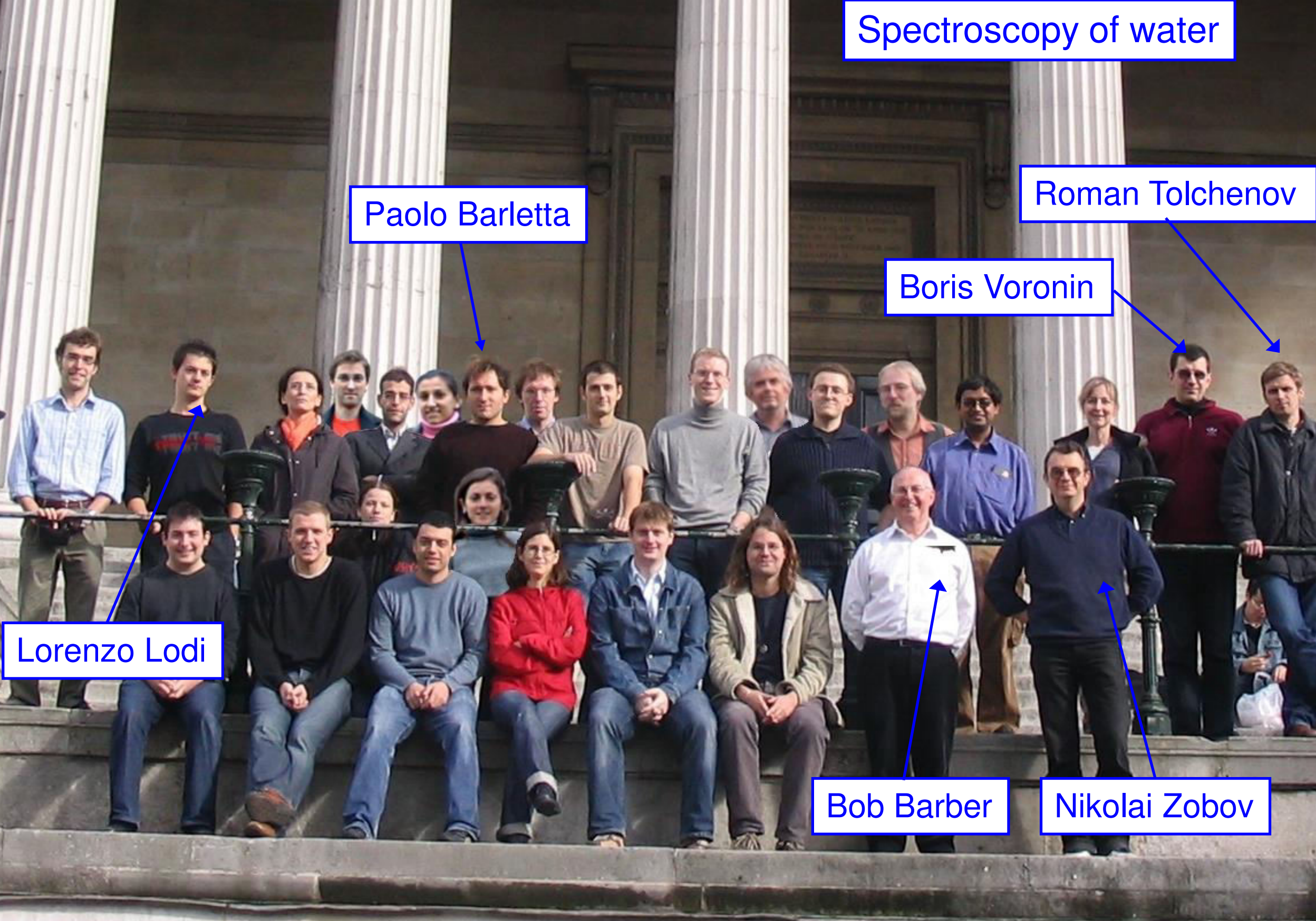




\section{ASTRONOMICAL SPECTROSCOPY}

An Introduction to the Atomic and

Molecular Physics of Astronomical Spectra

www.worldscibooks.com/physics/p371.html






\section{Labelling BT2 energy levels}

\begin{tabular}{|c|c|c|c|c|c|c|}
\hline $\mathrm{J}=25$ & $\mathrm{~J}=26$ & $\mathrm{~J}=27$ & $J=28$ & $\mathrm{~J}=29$ & $J=30$ & $\mathrm{~J}=31$ \\
\hline e & e & e & e & e & e & e \\
\hline 8 & 8 & 8 & 8 & 8 & 8 & 8 \\
\hline $6,171.595$ & $6,647.059$ & $7,139.12$ & $7,647.650$ & $8,172.487$ & $8,713.483$ & $9,270.484$ \\
\hline $7,026.716$ & $7,533.369$ & $8,055.92$ & $8,594.168$ & $9,147.872$ & $9,716.787$ & $10,300.639$ \\
\hline $7,715.449$ & $8,187.439$ & $8,675.83$ & $9,180.833$ & $9,702.386$ & $10,240.435$ & $10,794.934$ \\
\hline $7,729.146$ & $8,262.554$ & $8,811.35$ & $9,375.052$ & $9,953.431$ & $10,546.255$ & $11,153.286$ \\
\hline $8,297.771$ & $8,860.800$ & $9,437.28$ & $10,027.243$ & $10,630.630$ & $11,247.231$ & $11,876.321$ \\
\hline $8,668.232$ & $9,174.105$ & $9,695.15$ & $10,231.128$ & $10,781.645$ & $11,346.330$ & $11,925.255$ \\
\hline $8,679.039$ & $9,278.621$ & $9,892.85$ & $10,519.907$ & $11,158.558$ & $11,773.137$ & $12,333.738$ \\
\hline $9,041.386$ & $9,628.654$ & $10,200.59$ & $10,706.821$ & $11,230.920$ & $11,808.105$ & $12,468.284$ \\
\hline $9,240.960$ & $9,712.024$ & $10,236.40$ & $10,864.548$ & $11,512.638$ & $12,179.577$ & $12,748.026$ \\
\hline $9,417.429$ & $9,951.830$ & $10,500.80$ & $11,064.101$ & $11,641.403$ & $12,201.871$ & $12,836.462$ \\
\hline $9,560.232$ & $10,139.880$ & $10,658.50$ & $11,157.111$ & $11,671.777$ & $12,233.013$ & $12,865.287$ \\
\hline $9,709.826$ & $10,176.031$ & $10,737.08$ & $11,283.245$ & $11,799.695$ & $12,332.023$ & $12,880.080$ \\
\hline $9,830.647$ & $10,298.575$ & $10,782.82$ & $11,351.598$ & $11,983.277$ & $12,632.014$ & $13,297.793$ \\
\hline $10,003.358$ & $10,570.613$ & $11,150.02$ & $11,741.339$ & $12,341.648$ & $12,919.546$ & $13,487.796$ \\
\hline $10,147.816$ & $10,728.503$ & $11,301.88$ & $11,831.223$ & $12,376.627$ & $12,969.282$ & $13,596.429$ \\
\hline $10,283.872$ & $10,786.030$ & $11,325.91$ & $11,939.687$ & $12,569.512$ & $13,186.455$ & $13,758.743$ \\
\hline $10,380.255$ & $10,978.414$ & $11,558.74$ & $12,086.274$ & $12,628.900$ & $13,215.060$ & $13,876.022$ \\
\hline $10,549.891$ & $11,046.594$ & $11,593.38$ & $12,206.875$ & $12,751.210$ & $13,310.619$ & $13,884.951$ \\
\hline
\end{tabular}




\begin{tabular}{|c|c|c|c|c|c|c|c|c|c|c|c|c|c|}
\hline J=31 & & & & & & & $J=32$ & & & & & & \\
\hline 9270.484 & 0 & 0 & 0 & 31 & 1 & 31 & 9843.328 & 0 & 0 & 0 & 32 & 1 & 32 \\
\hline 10300.639 & 0 & 0 & 0 & 31 & 3 & 29 & 10899.132 & 0 & 0 & S & 32 & 3 & 30 \\
\hline 10794.935 & 0 & 1 & 0 & 31 & 1 & 31 & 11365.856 & 0 & 1 & 0 & 32 & 1 & 32 \\
\hline 11153.293 & 0 & 0 & 0 & 31 & 5 & 27 & 11774.277 & 0 & 0 & 0 & 32 & 5 & 28 \\
\hline 11876.839 & 0 & 0 & 0 & 31 & 7 & 25 & 12507.258 & 0 & 1 & S & 32 & 3 & 30 \\
\hline 11925.277 & 0 & 1 & 0 & 31 & 3 & 29 & 12528.734 & 0 & 0 & 0 & 32 & 7 & 26 \\
\hline 12333.738 & 0 & 2 & 0 & 31 & 1 & 31 & 12912.970 & 0 & 2 & 0 & 32 & 1 & 32 \\
\hline 12487.133 & 0 & 0 & 0 & 31 & 9 & 23 & 13151.952 & 0 & 0 & 0 & 32 & 9 & 24 \\
\hline 12748.027 & 1 & 0 & 0 & 31 & 1 & 31 & 13309.624 & 1 & 0 & 0 & 32 & 1 & 32 \\
\hline 12837.108 & 0 & 1 & 0 & 31 & 5 & 27 & 13443.559 & 0 & 0 & T & 32 & 0 & 32 \\
\hline 12880.079 & 0 & 0 & 1 & 31 & 0 & 31 & 13454.795 & 0 & 1 & 0 & 32 & 5 & 28 \\
\hline 13019.639 & 0 & 0 & 0 & 31 & 11 & 21 & 13697.050 & 0 & 0 & 0 & 32 & 11 & 22 \\
\hline 13487.823 & 0 & 2 & 0 & 31 & 3 & 29 & 14067.285 & 0 & 2 & 0 & 32 & 3 & 30 \\
\hline 13574.239 & 0 & 0 & 0 & 31 & 13 & 19 & 14236.728 & 0 & 1 & 0 & 32 & 7 & 26 \\
\hline 13598.867 & 0 & 1 & 0 & 31 & 7 & 25 & 14253.538 & 0 & 0 & 0 & 32 & 13 & 20 \\
\hline 13758.746 & 1 & 0 & 0 & 31 & 3 & 29 & 14345.561 & 1 & 0 & 0 & 32 & 3 & 30 \\
\hline 13884.951 & 0 & 0 & 1 & 31 & 2 & 29 & 14473.887 & 0 & 0 & 1 & 32 & 2 & 30 \\
\hline
\end{tabular}




\section{Room temperature $\mathrm{H}_{2}{ }^{16} \mathrm{O}$ lines}

- Strong line data about $9000 \mathrm{~cm}^{-1}$

- Compatability between mid and near infrared intensities

- Weak lines throughout whole spectrum

- Far infrared?

Solution strategy

largely experimental plus careful analysis? 


\section{Hot water (up to $\mathrm{T}=3000+\mathrm{K}$ )}

- New complete linelist available from UCL Accuracy?

- Experimental assignments

- New experiments?

- $\mathrm{H}_{2}{ }^{16} \mathrm{O}$ only?

(Some experiment for $\mathrm{HDO}$ and $\mathrm{D}_{2} \mathrm{O}$ )

- Line profiles?

Solution strategy:

largely theoretical with validation by experiment 


\section{Isotopologues}

- $\mathrm{H}_{2}{ }^{18} \mathrm{O}, \mathrm{H}_{2}{ }^{17} \mathrm{O}$, HDO lines patchy in visible

- $\mathrm{D}_{2}{ }^{16} \mathrm{O}$ not well known above $10000 \mathrm{~cm}^{-1}$

- Any interest in other isotopologues?

- Room T only?

- Line profiles?

Solution strategy

Isotopically enhanced experiments 


\section{Line profiles}

- Broadening by which species?

water, $\mathrm{O}_{2}, \mathrm{~N}_{2}$, air, $\mathrm{H}_{2}, \ldots \ldots$ ?

- T dependence?

- P dependence? (up to $10 \mathrm{~atm}$ ?)

Solution strategy

Theory validated by high quality experiment? 


\section{Validation}

- between experiments

- atmospheric spectra

- Theory vs experiment

- other 


\section{Distribution and storage}

- HITRAN

- Web database

eg Spectroscopic databank at Tomsk

- Publication or other means of distribution? 


\section{So what is the problem?}

Water is well studied $(30,000+$ lines in HITRAN)

\section{But}

- Water spectra have huge dynamic range

- Difficult to work with experimentally

- Spectra very dense: baseline hard to characterise

- Strong lines usually saturated (water-air spectra)

- Line profiles important (water-air \& water-water)

- Weak lines can be significant (pure water spectra)

- Line assignment difficult (Variational Methods) 


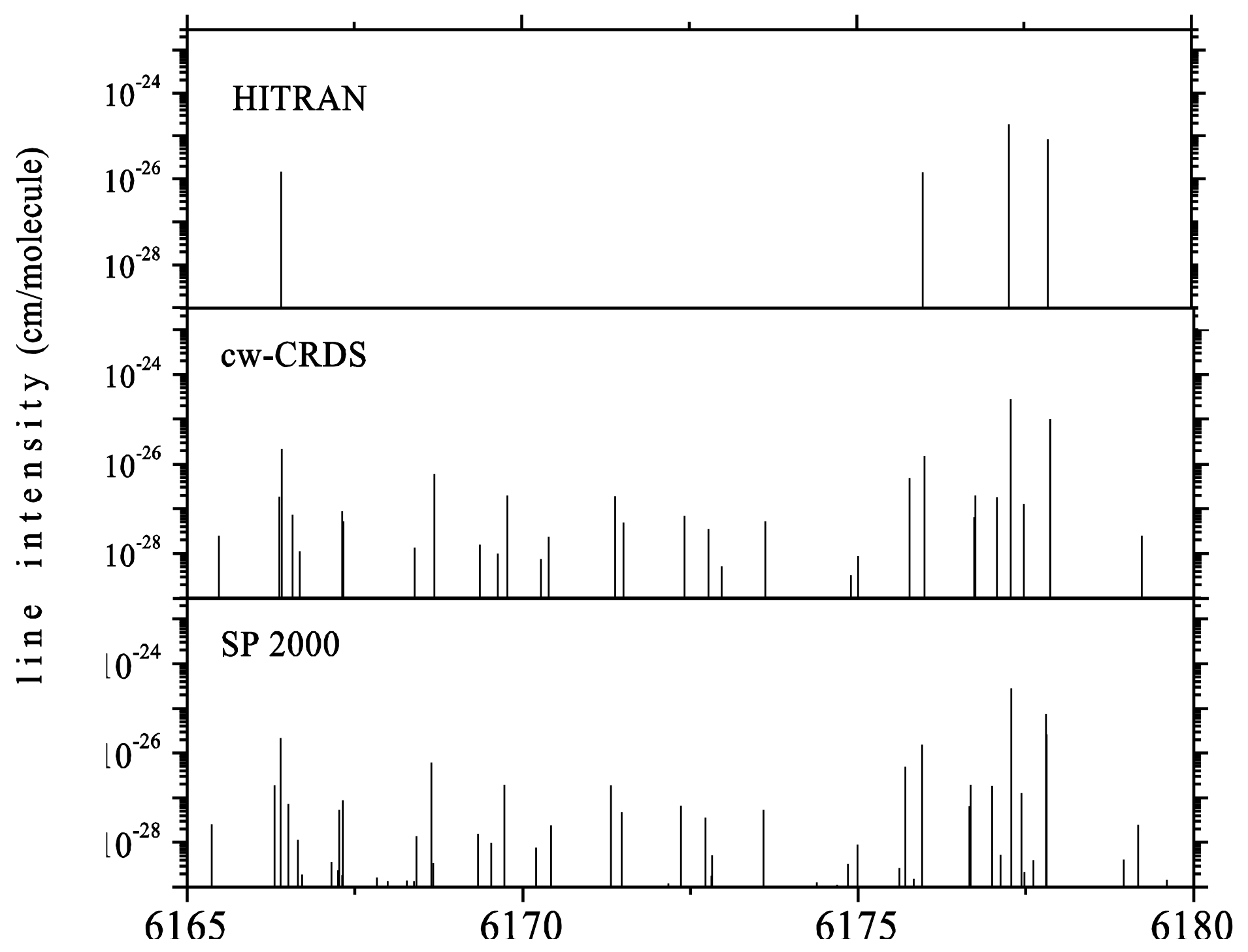




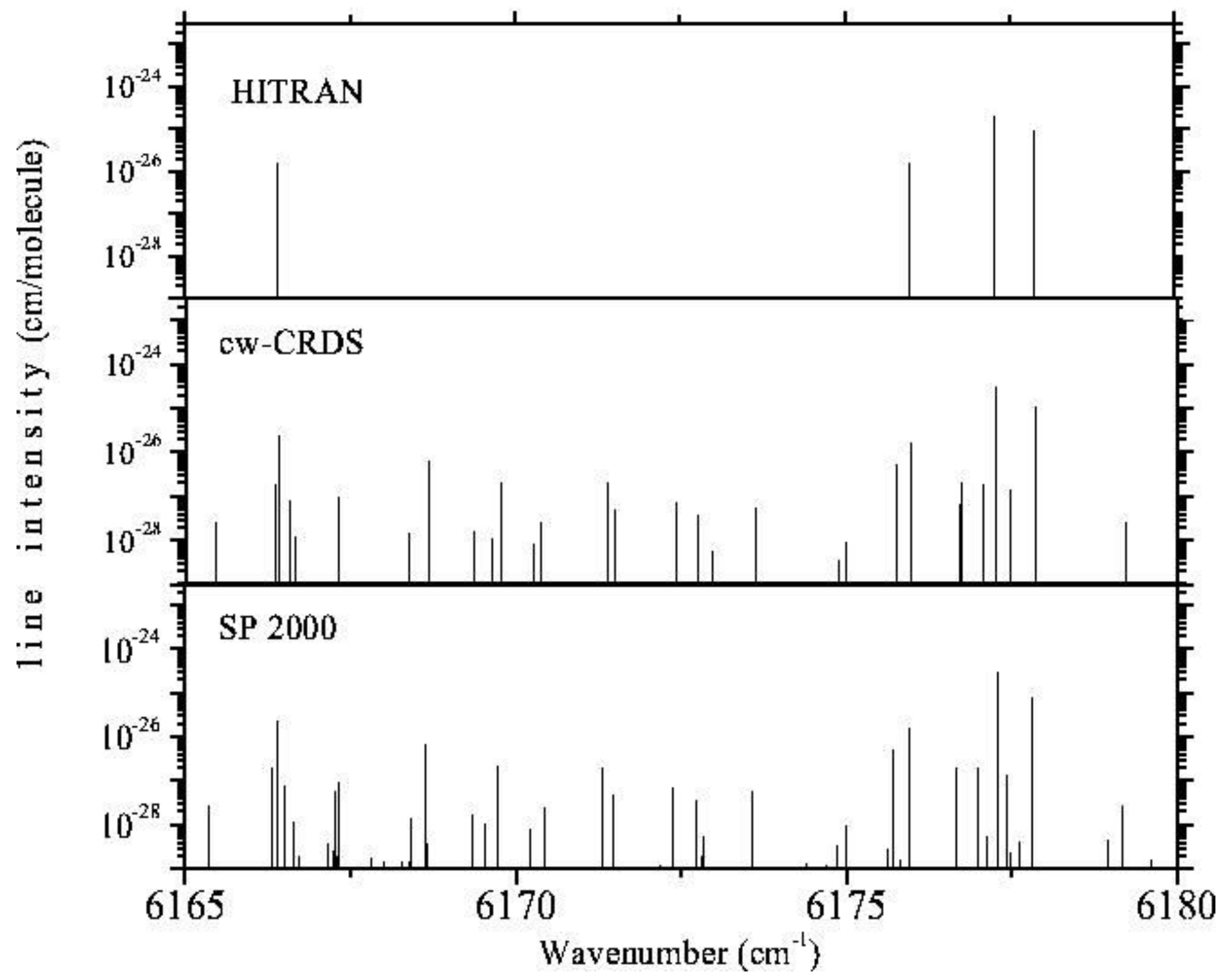




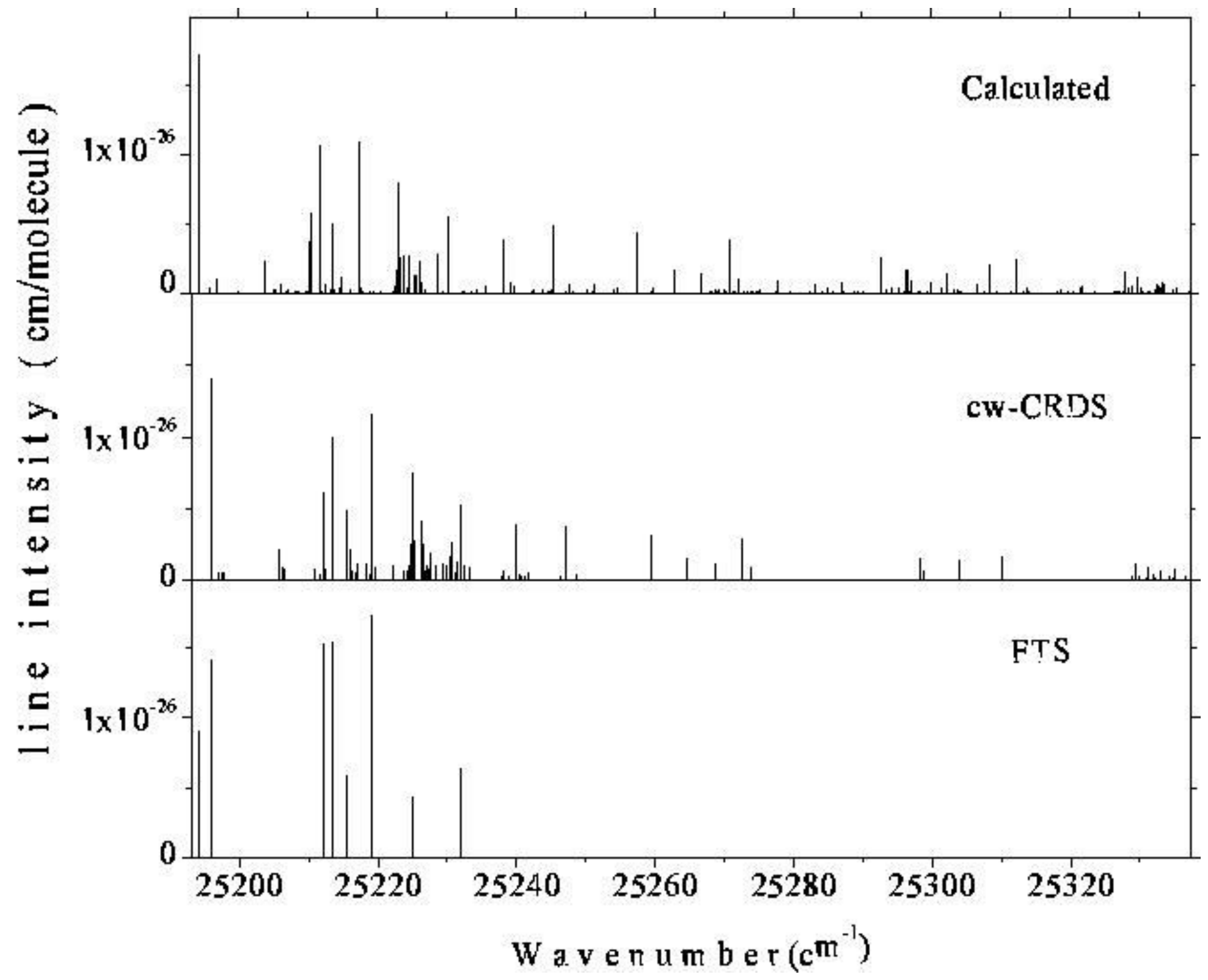

P. Dupre, T. Germain, A. Campargue, N.F. Zobov, O.L. Polyansky, S.V. Shirin, R.N. Tolchenov and J. Tennyson, J. Molec. Spectrosc. (to be submitted). 


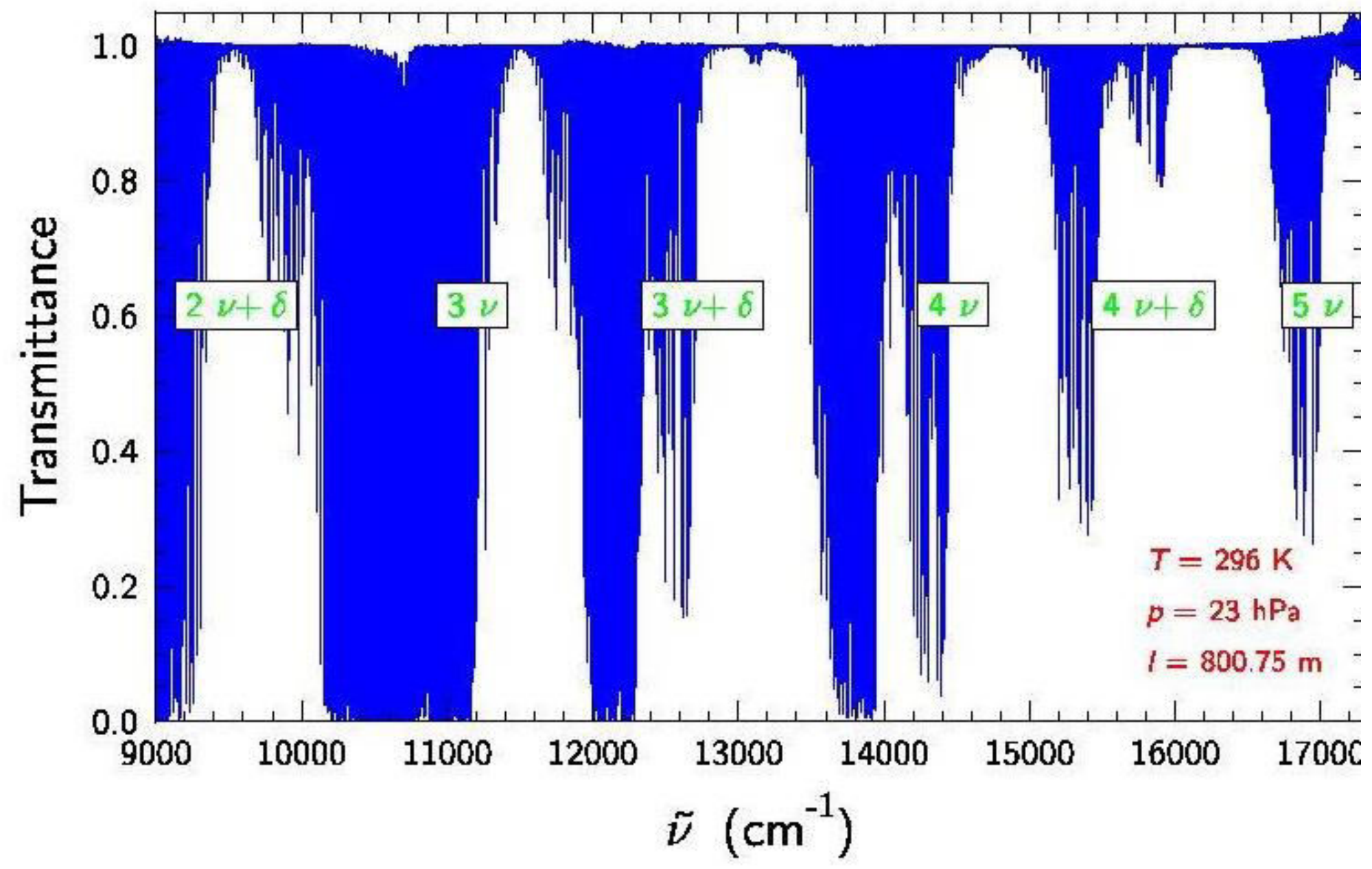




\section{$\mathrm{H}_{2} \mathrm{O}$ Visible Spectrum}

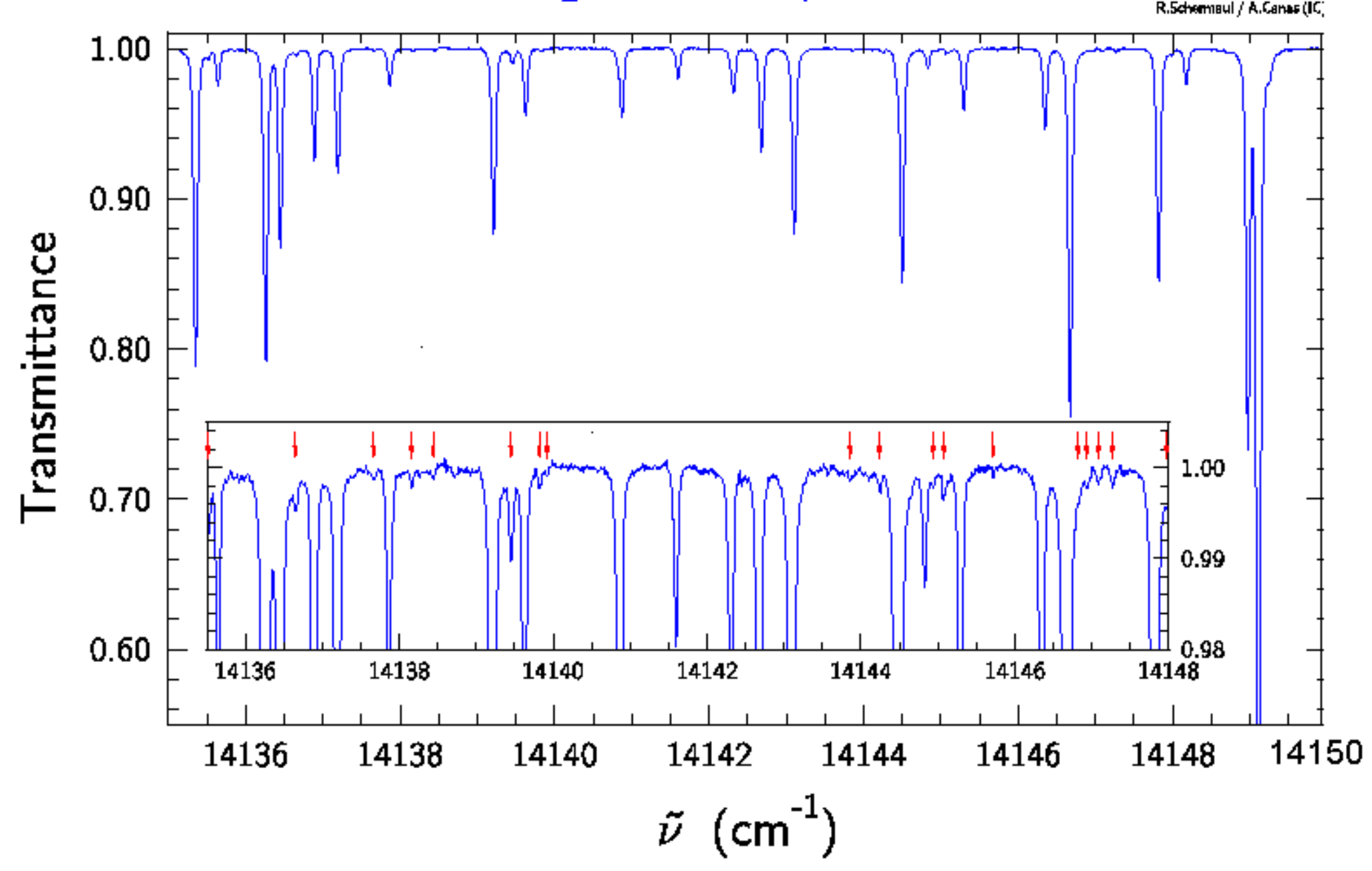




\section{Weak lines: new experimental measurements}

Weak water lines Very difficult to record

Only a few weak lines in HITRAN

MSF data (NERC) : 8m cell, pure water vapour

- Schermaul, Learner et al.

- Bruker F.T.S.

- Range : 9000-12 $700 \mathrm{~cm}^{-1}$

- $\mathrm{T}: 295.7 \mathrm{~K}$

- $\mathrm{p}\left(\mathrm{H}_{2} \mathrm{O}\right): 22.93 \mathrm{hPa}$

- pathlength $~ 800.8 \mathrm{~m}$

- Number of lines : 7923

- Number of new lines : 1082
- Schermaul, Learner et al.

- Bruker F.T.S.

- Range :11 700-14 $750 \mathrm{~cm}^{-1}$

- $\mathrm{T}: 294.4 \mathrm{~K}$

- $\mathrm{p}\left(\mathrm{H}_{2} \mathrm{O}\right): 23.02 \mathrm{hPa}$

- pathlength $\sim 800.8 \mathrm{~m}$

- Number of lines : 5316

- Number of new lines : 1534 Also data in $6000-9000 \mathrm{~cm}^{-1}$ region 


\section{Weak lines: new experimental measurements}

REIMS data, $50 \mathrm{~m}$ cell, pure water vapor (also water-air)

- Coheur et al., Fally et al.

- Bruker F.T.S

- Range :13000 - $25020 \mathrm{~cm}^{-1}$

- $\mathrm{T}: 291.3 \mathrm{~K}$

- $\mathrm{p}\left(\mathrm{H}_{2} \mathrm{O}\right): 18.32 \mathrm{hPa}$

- pathlength $\sim 602.32 \mathrm{~m}$

- Number of lines: 9353

- Number of new lines : 2286
- Merienne et al.

- Bruker F.T.S

- Range : 9250 - $13000 \mathrm{~cm}^{-1}$

- $\mathrm{T}: 292 \mathrm{~K}$

- $\mathrm{p}\left(\mathrm{H}_{2} \mathrm{O}\right): 23.02 \mathrm{hPa}$

- pathlength $\sim 602.32 \mathrm{~m}$

- Number of lines : 7061

- Number of new lines : small 


\begin{tabular}{|c|c|c|c|c|c|c|c|}
\hline \multicolumn{2}{|c|}{ Band } & \multirow{3}{*}{$\nu_{0}\left(\mathrm{~cm}^{-1}\right)$} & \multicolumn{2}{|c|}{ Previoux } & \multicolumn{3}{|c|}{ This work } \\
\hline local & uormal & & lines & levels & lin & $\operatorname{les}$ & levels \\
\hline & & & & & cxl & cal & \\
\hline$(3,0)+4$ & 340 & 16534 & & & 33 & 15 & 26 \\
\hline$(3,0)-4$ & 241 & 16546.324 & & & 42 & 16 & 33 \\
\hline$(2,1)+4$ & 142 & 16795 & & & 76 & 4 & 33 \\
\hline$(4,0)-2$ & 321 & 16821.635 & 131 & 54 & 34 & & 3 \\
\hline$(4,0)+2$ & 420 & 16823.321 & 39 & 22 & 63 & 9 & 26 \\
\hline$(5,0)+0$ & 500 & 16898.271 & 114 & 56 & 66 & 2 & 8 \\
\hline$(5,0)-0$ & 401 & 16898.842 & 203 & 76 & 50 & & \\
\hline$(2,1)-4$ & 043 & 16967 & & & 8 & 8 & 11 \\
\hline$(3,1)+2$ & 222 & 17227 & 2 & 2 & 38 & 12 & 26 \\
\hline$(3,1)-2$ & 123 & 17312.539 & 53 & 32 & 18 & 2 & 6 \\
\hline$(4,1)+0$ & 302 & 17458.354 & 88 & 43 & 48 & 3 & 8 \\
\hline$(4,1)-0$ & 203 & 17495.528 & 137 & 57 & 72 & & 4 \\
\hline$(3,2)+0$ & 104 & 17748.134 & 5 & 4 & 48 & 25 & 41 \\
\hline$(4,0)-3$ & 331 & 18265.819 & 52 & 28 & 60 & 6 & 22 \\
\hline$(4,0)+3$ & 430 & & 2 & 1 & 6 & & 3 \\
\hline$(2,1)-5$ & 053 & 18350 & & & 18 & 3 & 11 \\
\hline$(5,0)-1$ & 411 & 18393.314 & 62 & 27 & 71 & 2 & 24 \\
\hline$(5,0)+1$ & 510 & 18392.974 & 15 & 7 & 50 & 4 & 27 \\
\hline$(3,1)-3$ & 133 & 18758.634 & & & 24 & 13 & 24 \\
\hline$(2,2)+3$ & 034 & 18977 & & & 10 & 3 & 8 \\
\hline$(5,0)-1$ & 411 & 18989.961 & 20 & 11 & 66 & 8 & 34 \\
\hline$(4,0)-4$ & 341 & 19679.196 & 6 & 4 & 54 & 6 & 32 \\
\hline$(2,1)-6$ & 063 & 19720 & & & 6 & & 3 \\
\hline$(6,0)-0$ & 501 & 19781.105 & 88 & 39 & 59 & 2 & 15 \\
\hline$(6,0)+0$ & 600 & 19782 & 29 & 17 & 51 & 5 & 18 \\
\hline$(5,0)-2$ & 421 & 19863 & & & 19 & 8 & 16 \\
\hline$(5,0)+2$ & 520 & 19864 & & & 27 & 1 & 11 \\
\hline$(4,1)-2$ & 223 & 20441.882 & & & 20 & 5 & 11 \\
\hline$(5,1)+0$ & 402 & 20534 & & & 23 & 15 & 25 \\
\hline$(5,1)-0$ & 303 & 20543.137 & 25 & 14 & 32 & 7 & 18 \\
\hline$(6,0)-1$ & 511 & 21221.828 & 25 & 11 & 21 & 1 & 11 \\
\hline$(6,0)+1$ & 610 & 21221.569 & 9 & 6 & 4 & 2 & 4 \\
\hline (5n-3 & 131 & 21312 & & & 16. & 2 & 11 \\
\hline
\end{tabular}

M. Carleer, A. Jenouvrier, A.-C. Vandaele, P.F. Bernath, M.F. Marienne, R. Colin, N.F. Zobov, O.L. Polyansky, J. Tennyson \& V.A. Savin J. Chem. Phys., 111, 2444 (1999) 
MSF spectra: line parameter retrieval using GOBLIN

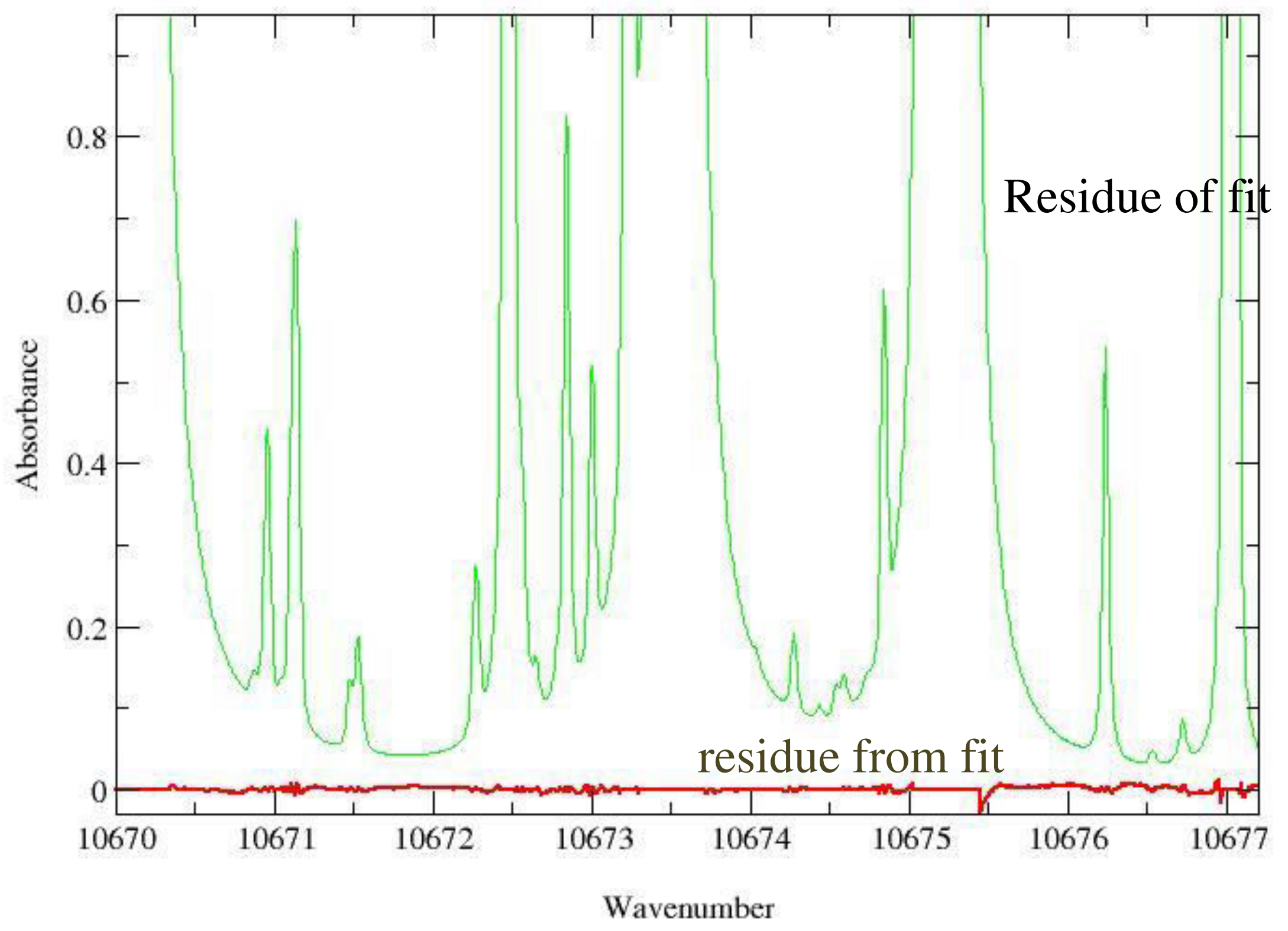




\section{Intensity comparison for weak lines: MSF vs Rheims}

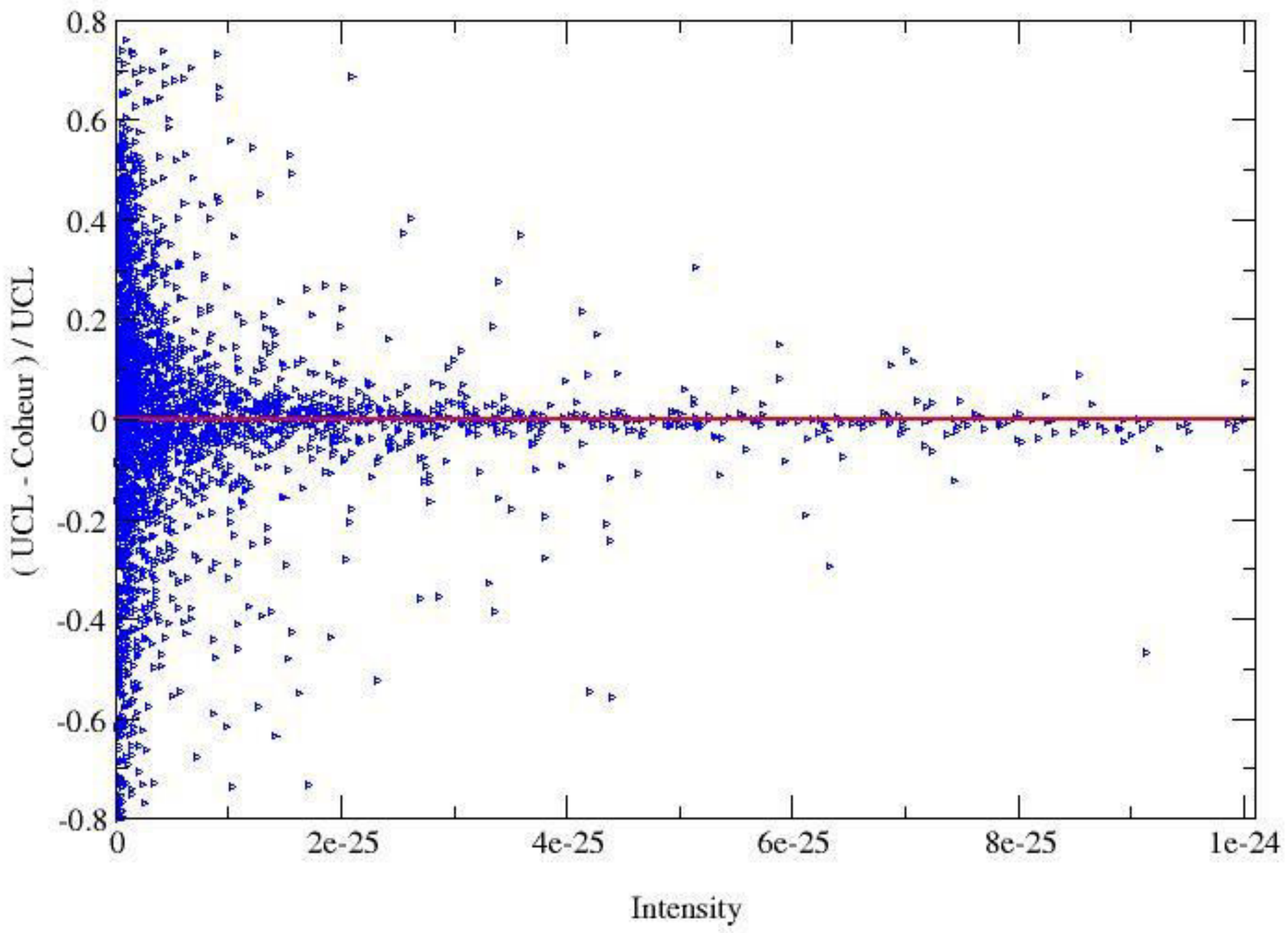




\section{Reliable intensities required for satellite retrievals}

MSF data (ESA) : 8m cell, water-air spectra

- Schermaul, Learner, Brault, Newnham et al.

- Bruker F.T.S.

- Range : 9000 - $12700 \mathrm{~cm}^{-1}$

- $\mathrm{T}: 295.7 \mathrm{~K}$ (also $253 \mathrm{~K}$ )

- $\mathrm{p}\left(\mathrm{H}_{2} \mathrm{O}\right): 10.03 \mathrm{hPa}$

- Pathlength: SPAC $4.938 \mathrm{~m}$

$$
\text { LPAC } 32.75 \mathrm{~m}, 128.75 \mathrm{~m}, 512.75 \mathrm{~m}
$$

- Number of lines : 7923

- Number of new lines : 1082 


\section{Intensity data compared to HITRAN-96 by polyad for spectral region $8500-15800 \mathrm{~cm}^{-1}$}

\begin{tabular}{|l|c|c|c|c|}
\hline Polyad & $\begin{array}{l}\text { Integrated } \\
\text { absorbance }\end{array}$ & $\begin{array}{l}\text { Spectral } \\
\text { linefits }\end{array}$ & $\begin{array}{l}\text { Ab Initio } \\
\text { calculation }\end{array}$ & $\begin{array}{c}\text { Correction } \\
\text { Giver et al. }\end{array}$ \\
\hline $2 v+\delta$ & & 1.26 & 1.31 & 0.92 \\
\hline $3 v$ & 1.19 & 1.21 & 1.04 & 1.14 \\
\hline $3 v+\delta$ & 1.26 & 1.25 & 1.25 & 1.09 \\
\hline $4 v$ & & 1.06 & 1.04 & 0.96 \\
\hline
\end{tabular}

Numbers are ratio of total intensity to Hitran96 HITRAN underestimates intensity of strong lines!

D Belmiloud et al, Geophys. Res. Lett., 27, 3703 (2000). 
Intensity comparison for strong lines: ESA vs Hitran 2000

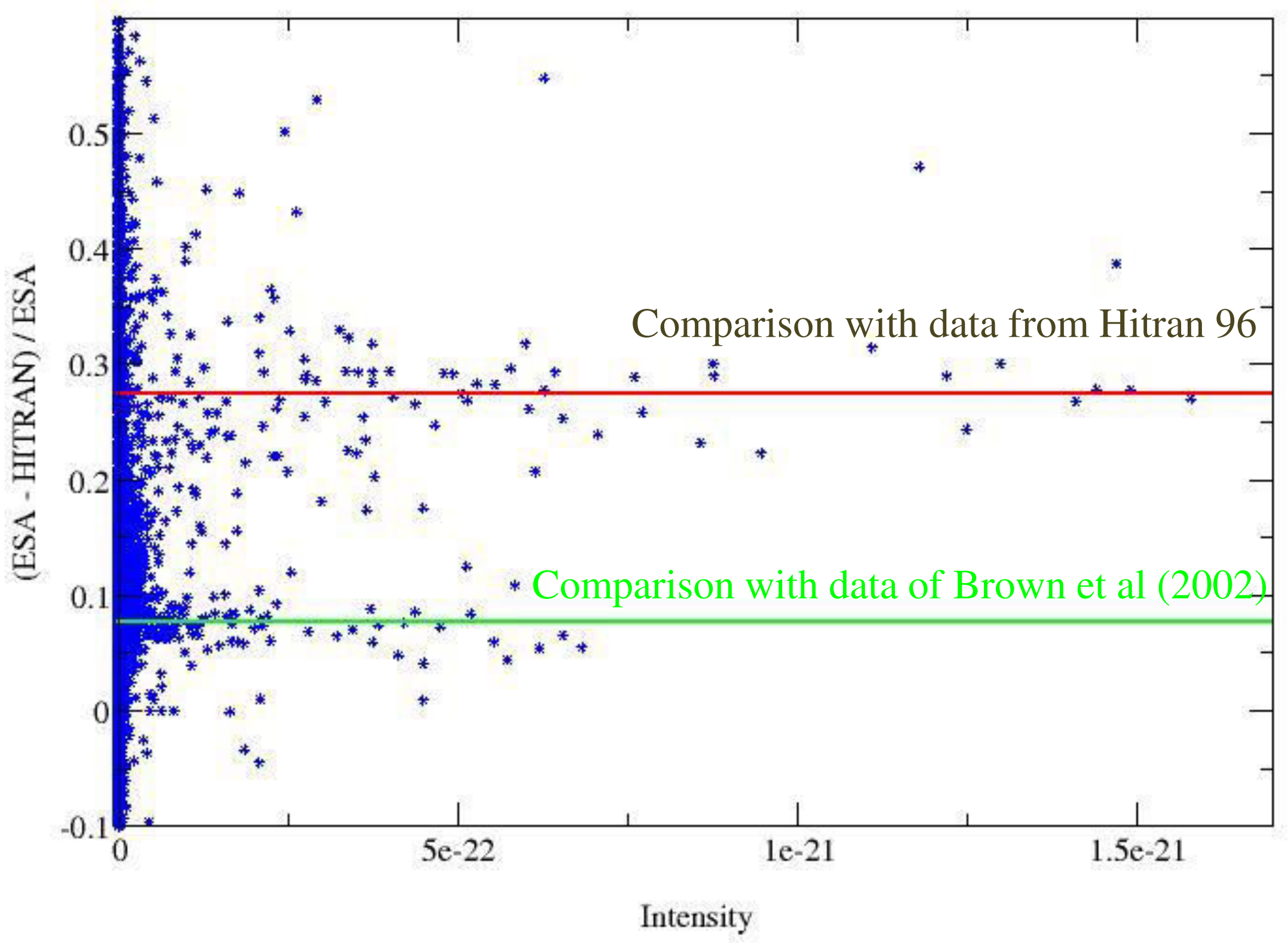


ESA spectra: line parameter retrieval

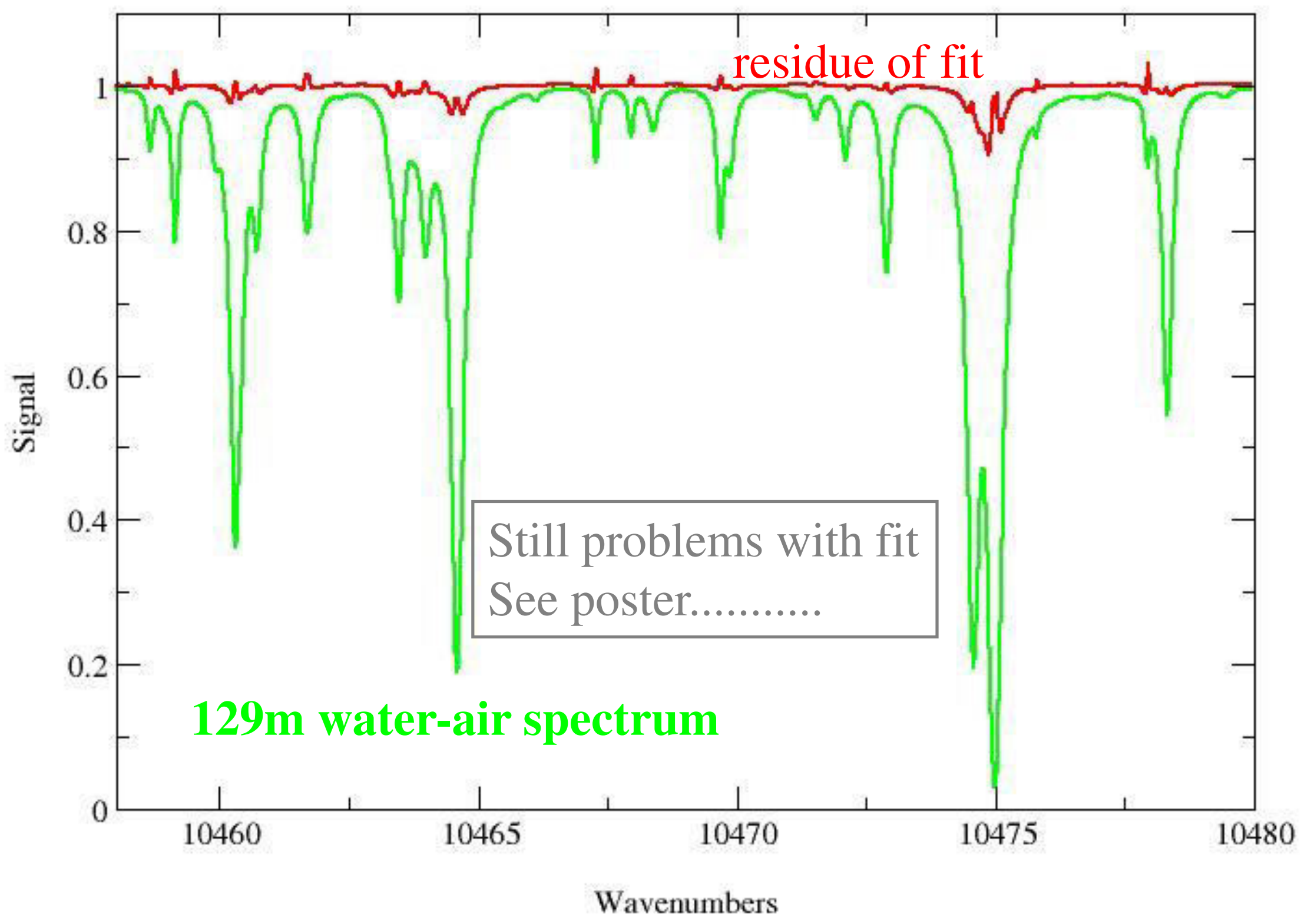


Validation using atmospheric spectra
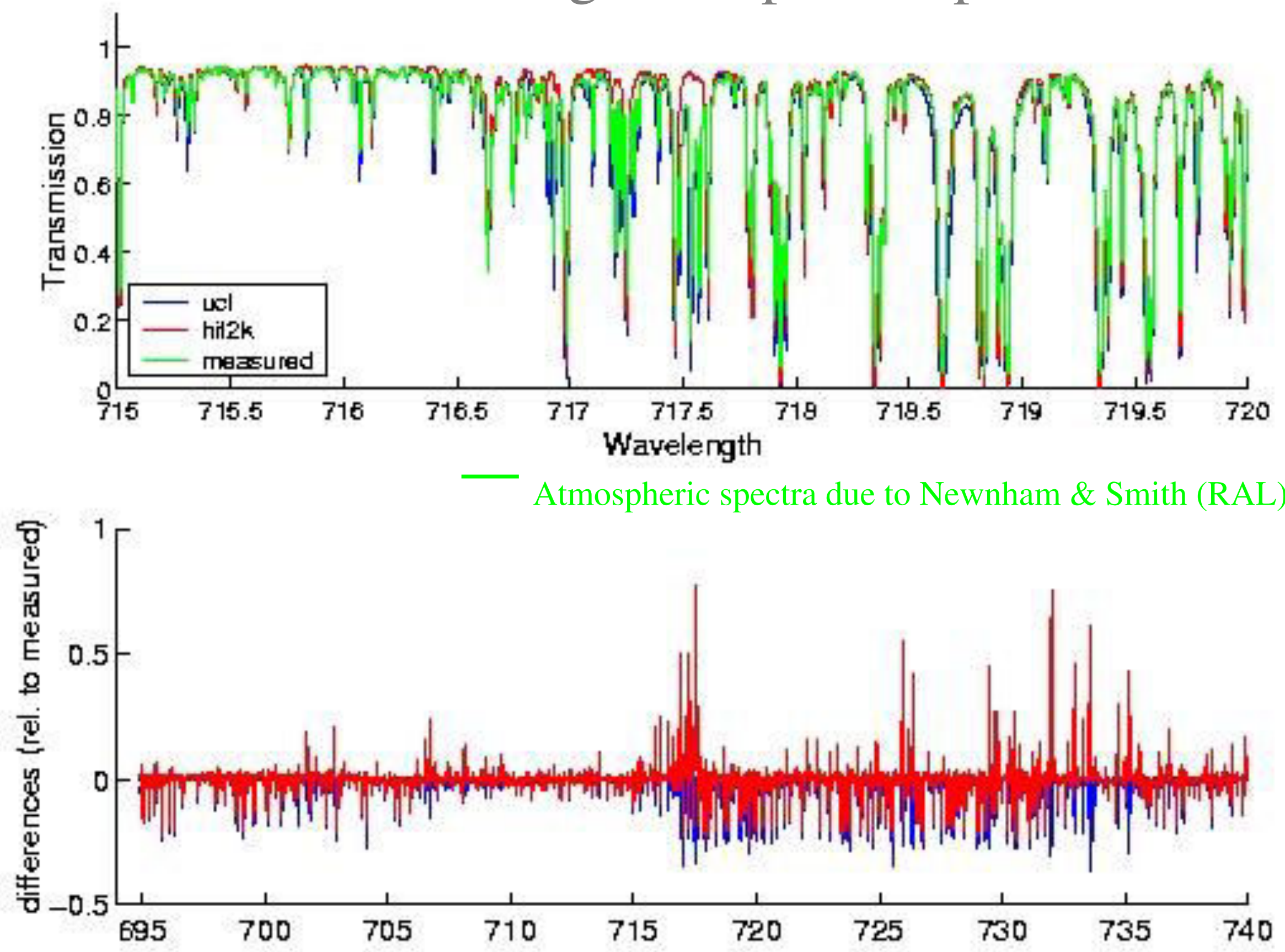


\section{Water isotopmers in the visible}

- Fourier transform spectra in Kitt Peak archive up to $15000 \mathrm{~cm}^{-1}$

$\mathrm{H}_{2}{ }^{18} \mathrm{O}$ : M. Tanaka, J.W. Brault and J. Tennyson, J. Molec. Spectrosc., 216, 77 (2002).

$\mathbf{H}_{2}{ }^{17} \mathrm{O}$ : M. Tanaka, O. Naumenko, J.W. Brault and J. Tennyson to be published

- Cavity ringdown spectra from Amsterdam about $17000 \mathrm{~cm}^{-1}$

$\mathbf{H}_{2}{ }^{18}$ O: M. Tanaka, M. Sneep, W. Ubachs \& J. Tennyson, J. Molec. Spectrosc., 226, 1 (2004).

$\mathrm{H}_{2}{ }^{17} \mathrm{O}$ : being analysed at $\mathrm{UCL}$

- HDO: Brussels/Rheims spectra of Coheur et al being analysed in Tomsk 


\section{Missing absorption due to water:}

\section{First estimates}

- In the red and visible :

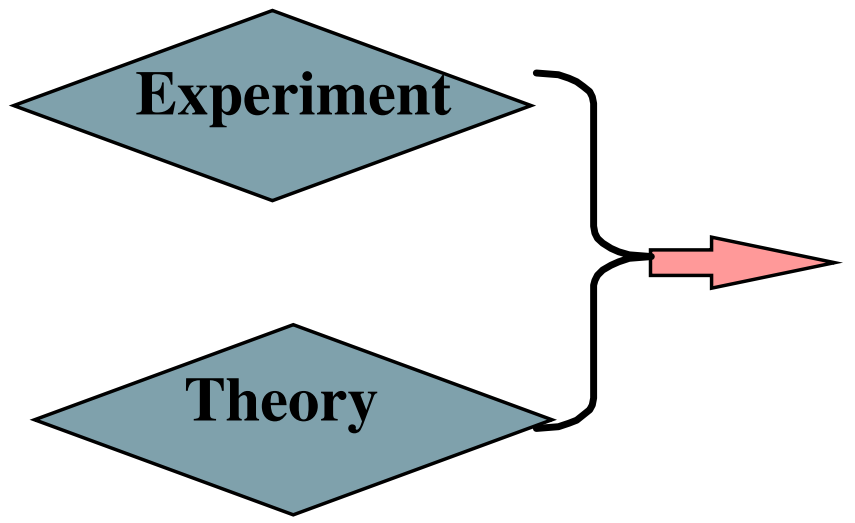

\section{Radiative Transfer} Model
Atmospheric

absorption

- Unobserved weak lines have a significant effect : $~ 3 \mathbf{W m}^{-2}$

$>$ Estimated additional 2.5-3 \% absorption in the near I.R/Red.

$>$ Estimated additional 8-11 \% absorption in the 'Blue'?

- Underestimate of strong lines even more important : $\sim 8 \mathrm{Wm}^{-2}$

$>$ Estimated additional $8 \%$ absorption in the near I.R/Red. 


\section{Missing absorption due to water: Outstanding issues}

- In the near infrared and red:

$>$ Contributions due to $\mathrm{H}_{2}{ }^{18} \mathrm{O}, \mathrm{H}_{2}{ }^{17} \mathrm{O}$ and HDO.

$>$ Possible role of water dimer $\left(\mathrm{H}_{2} \mathrm{O}\right)_{2}$.

- In the blue and ultraviolet:

$>$ Are $\mathrm{H}_{2}{ }^{16} \mathrm{O}$ line intensities also underestimated?

$>$ Contribution due to weak lines 